Article

\title{
Protein Tyrosine Phosphatase 1B Inhibitors from the Stems of Akebia quinata
}

\author{
Jin-Pyo An ${ }^{1}$, Thi Kim Quy Ha ${ }^{1}$, Jinwoong Kim ${ }^{1}$, Tae Oh $\mathrm{Cho}^{2}$ and Won Keun Oh ${ }^{1, *}$ \\ 1 Korea Bioactive Natural Material Bank, Research Institute of Pharmaceutical Sciences, College of Pharmacy, \\ Seoul National University, Seoul 151-742, Korea; ntopjp77@gmail.com (J.-P.A.); \\ htkquy@ctu.edu.vn (T.K.Q.H.); jwkim@snu.ac.kr (J.K.) \\ 2 Marine Bio Research Center, Department of Life Science, Chosun University, Gwangju 501-759, Korea; \\ tocho@chosun.ac.kr \\ * Correspondence: wkoh1@snu.ac.kr; Tel.: +82-2-880-7872
}

Academic Editor: Isabel C. F. Ferreira

Received: 17 July 2016; Accepted: 10 August 2016; Published: 19 August 2016

\begin{abstract}
PTP1B deficiency in mouse mammary tumor virus (MMTV)-NeuNT transgenic mice inhibited the onset of MMTV-NeuNT-evoked breast cancer, while its overexpression was observed in breast cancer. Thus, PTP1B inhibitors are considered chemopreventative agents for breast cancer. As part of our program to find PTP1B inhibitors, one new diterpene glycoside (1) and 13 known compounds (2-14) were isolated from the methanol extract of the stems of Akebia quinata. All isolates were identified based on extensive spectroscopic data analysis, including UV, IR, NMR and MS. Compounds 2, 3, 6, 8 and 11 showed significant inhibitory effects on the PTP1B enzyme, with $\mathrm{IC}_{50}$ values ranging from $4.08 \pm 1.09$ to $21.80 \pm 4.74 \mu \mathrm{M}$. PTP1B inhibitors also had concentration-dependent cytotoxic effects on breast cancer cell lines, such as MCF7, MDA-MB-231 and tamoxifen-resistant MCF7 (MCF7/TAMR) ( $\mathrm{IC}_{50}$ values ranging from $0.84 \pm 0.04$ to $7.91 \pm 0.39 \mu \mathrm{M}$ ). These results indicate that compounds $\mathbf{6}$ and $\mathbf{8}$ from Akebia quinata may be lead compounds acting as anti-breast cancer agents.
\end{abstract}

Keywords: Akebia quinata; terpenes; protein tyrosine phosphatase 1B (PTP1B); breast cancer

\section{Introduction}

Breast cancer is a threat to the health of women, ranking as the most common cancer in women globally, with more than 1,600,000 cases diagnosed in 2012 [1]. The American Association for Cancer Research reported that the number of breast cancer cases in the United States will increase by 50 percent in 2030 compared to 2011. Furthermore, the cost for care of breast cancer was the highest among all cancer types in the USA in 2010 at $\$ 16.5$ billion [2]. Although several drugs, including adriamycin and taxotere, are used to treat breast cancer, many adverse effects, such as heart problems and reduction of white blood cell numbers, have been reported [1]. Thus, the discovery of anti-breast cancer agents is an urgent need.

Protein tyrosine phosphatase 1B (PTP1B), a negative insulin regulator, has been implicated in the signaling of breast cancer. PTP1B overexpression was observed in $72 \%$ of breast tumors compared to normal epithelium, and 38\% of tumors showed maximal expression of PTP1B [3]. PTP1B siRNA-transfected cells significantly prevented insulin-like growth factor 2 (IGF-2)-induced MCF-cell migration, and the growth of MCF-7 cells was delayed when PTP1B was inhibited [4]. When PTP1B knockout mice were crossed with mouse mammary tumor virus (MMTV)-NeuNT mice, less than $40 \%$ of MMTV-NeuNT/PTP1 ${ }^{-/-}$mice developed breast tumors over a 3 year period, whereas MMTV-NeuNT/PTP1B ${ }^{+/+}$mice had tumors with an average latency of 13 months [5]. 
PTP1B is also known as a novel target for acting against type 2 diabetes, not only against breast cancer. PTP1B is expressed ubiquitously in all insulin-responsive tissue and negatively regulates the insulin cascade. PTP1B knockout mice had enhanced insulin sensitivity and glucose homeostasis, preventing type 2 diabetes [6]. Insulin-induced phosphorylation at both the insulin receptor $\beta$-chain (IR $\beta$ ) and insulin-receptor substrate-1 (IRS-1) was improved in free fatty acid (FFA)-insulin-induced hepatocyte model cells when PTP1B was reduced [7]. Therefore, finding a leading molecule for inhibiting PTP1B might help prevent the prevalence of not only breast cancer but also diabetes and obesity.

The stems of Akebia quinata (Hoult) Decne. were investigated for PTP1B inhibitory effects and cytotoxic activity. Previous phytochemical studies identified some of the constituents of A. quinata as triterpene glycosides, triterpenes, lignans, and phenylethanoid glycosides [8]. The biological activities of its dried stem have been reported to include anti-inflammatory, anti-obesity and anti-oxidant effects [9]. However, PTP1B inhibitory activity from this plant has not been reported. In this paper, we report one new diterpene glycoside (1) and 13 known compounds, including four diterpenes (2-5). Among them, compounds 2, 3, 6, 8 and $\mathbf{1 1}$ exhibited significant PTP1B inhibitory activity. Furthermore, the inhibitory activities of the isolated compounds were examined against MCF-7, MDB-MB-231 and tamoxifen-resistant MCF7 (MCF7/TAMR) breast cancer cell lines.

\section{Results}

The EtOAc soluble fraction exhibited potential inhibitory activity of PTP1B at $20 \mu \mathrm{g} / \mathrm{mL}$ in a PTP enzyme assay. Activity-guided fractionations of EtOAc soluble fraction were performed by successive chromatographic procedures, including silica gel chromatography, Sephadex LH-20, RP- $\mathrm{C}_{18}$ and HPLC to afford one new diterpene glycoside (1) and 13 known compounds (2-14) (Figure 1).

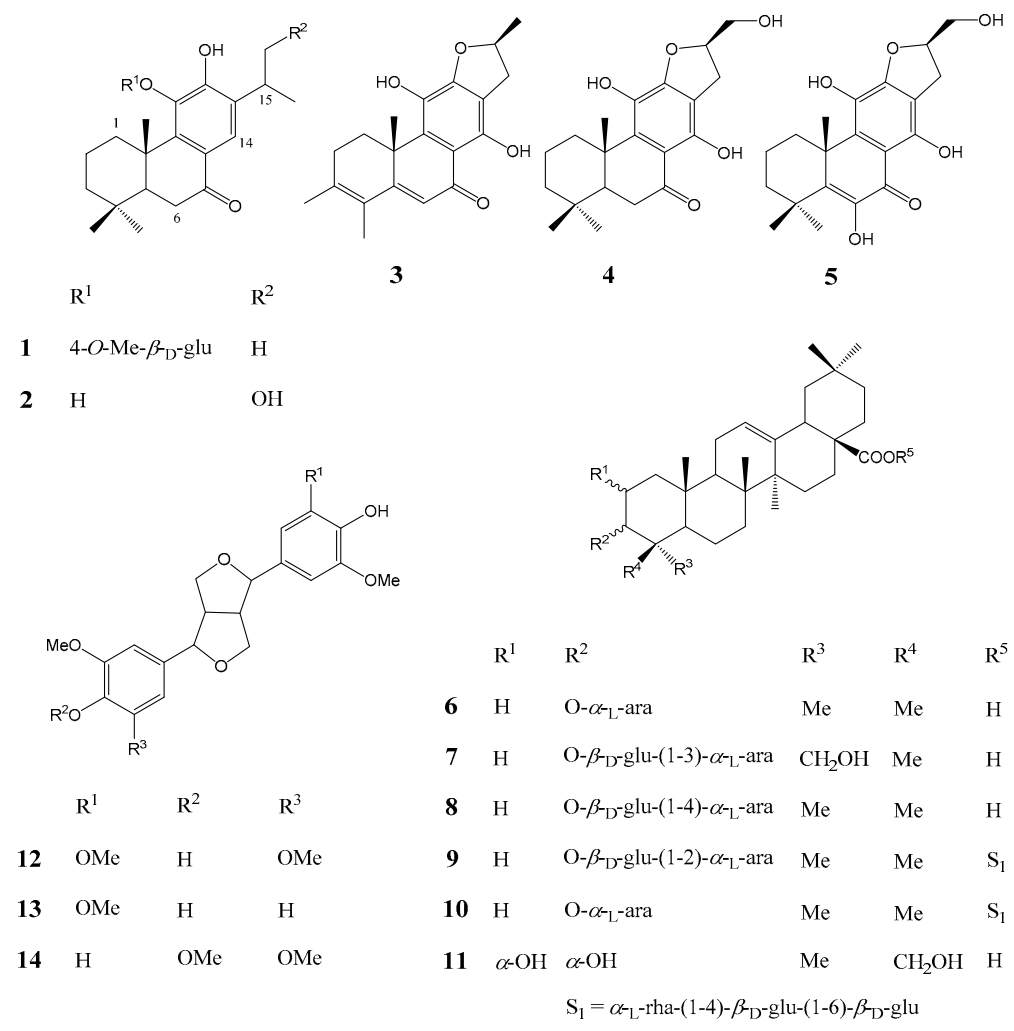

Figure 1. Chemical structures of compounds 1-14 isolated from the stems of Akebia quinata.

Compound 1, obtained as a yellowish gum, has a molecular formula of $\mathrm{C}_{27} \mathrm{H}_{40} \mathrm{O}_{8}$ as established by a quasimolecular ion in the HRESIMS at $m / z 493.2782[\mathrm{M}+\mathrm{H}]^{+}$(calcd. for $\mathrm{C}_{27} \mathrm{H}_{41} \mathrm{O}_{8}, 493.2796$ ). 
The IR spectrum of compound 1 suggested the presence of hydroxyl $\left(3287 \mathrm{~cm}^{-1}\right)$, carbonyl $\left(1666 \mathrm{~cm}^{-1}\right)$ and phenyl $\left(1593 \mathrm{~cm}^{-1}\right)$ functionalities. The ${ }^{1} \mathrm{H}-\mathrm{NMR}$ spectrum showed signals for five methyl groups $\left[\delta_{\mathrm{H}} 1.20(3 \mathrm{H}, \mathrm{d}, J=6.9 \mathrm{~Hz}, \mathrm{H}-16), 1.23(3 \mathrm{H}, \mathrm{d}, J=6.9 \mathrm{~Hz}, \mathrm{H}-17), 0.93(3 \mathrm{H}, \mathrm{s}, \mathrm{H}-18), 1.02(3 \mathrm{H}, \mathrm{s}, \mathrm{H}-19)\right.$ and $1.57(3 \mathrm{H}, \mathrm{s}, \mathrm{H}-20)]$, one aromatic single proton $\left(\delta_{\mathrm{H}} 7.73,1 \mathrm{H}, \mathrm{s}, \mathrm{H}-14\right)$ and one methine proton $\left(\delta_{\mathrm{H}} 3.27,1 \mathrm{H}, \mathrm{m}, \mathrm{H}-15\right)$ (Figure $\left.\mathrm{S} 1\right)$. The ${ }^{13} \mathrm{C}-\mathrm{NMR}$ spectrum revealed the presences of ketone carbon at $\delta_{C} 201.7$ and one methoxy at $\delta_{C} 60.8$ (Figure S2). The location of the ketone group at $C-7$ was confirmed by the HMBC correlations from $\mathrm{H}-14\left(\delta_{\mathrm{H}} 7.73\right), \mathrm{H}-6 \alpha\left(\delta_{\mathrm{H}} 2.61\right)$ and $\mathrm{H}-6 \beta\left(\delta_{\mathrm{H}} 2.58\right)$ to $\mathrm{C}-7\left(\delta_{\mathrm{C}} 201.7\right)$ (Figure S3). The presence of a hydroxyl group at $\mathrm{C}-12$ was confirmed based on the HMBC correlations from $\mathrm{H}-14\left(\delta_{\mathrm{H}} 7.73\right)$ to $\mathrm{C}-12\left(\delta_{\mathrm{C}} 154.6\right)$ and from $\mathrm{H}-15\left(\delta_{\mathrm{H}} 3.27\right)$ to $\mathrm{C}-12\left(\delta_{\mathrm{C}} 154.6\right)$. One aromatic proton at C-14 was deduced by HMBC correlations from a single proton at $\delta_{\mathrm{H}} 7.73$ to C-7 $\left(\delta_{\mathrm{C}} 201.7\right), \mathrm{C}-9$ $\left(\delta_{C} 148.5\right), C-11\left(\delta_{C} 143.8\right)$ and $C-12\left(\delta_{C} 154.6\right)$ (Figure 2 and Figures $\left.S 4-S 7\right)$. The ${ }^{1} \mathrm{H}$ - and ${ }^{13} \mathrm{C}-\mathrm{NMR}$ data suggested that compound 1 resembled with those of sugiol structure except for the presence of one additional sugar at the $C-11$ position. The $\beta$-glucopyranosyl unit at $C-11$ of compound 1 was confirmed by the HMBC correlation from the anomeric proton $\left(\delta_{\mathrm{H}} 4.54,1 \mathrm{H}, \mathrm{d}, J=7.7 \mathrm{~Hz}\right)$ to $\mathrm{C}-11$ $\left(\delta_{C} 143.8\right)$. The presence of one methyl group at $C-4^{\prime}\left(\delta_{C} 80.3\right)$ of glucopyranosyl was also suggested by HMBC correlations from the methoxy $\left(\delta_{\mathrm{H}} 3.58,3 \mathrm{H}\right)$ to $\mathrm{C}-4^{\prime}\left(\delta_{\mathrm{C}} 80.3\right)$ (Figure 2). Thus, compound 1 was established as 11-O-(4-O-methyl- $\beta$-D-glucopyranosyl)-12-hydroxyabieta-8,11,13-triene-7-one (Table 1).

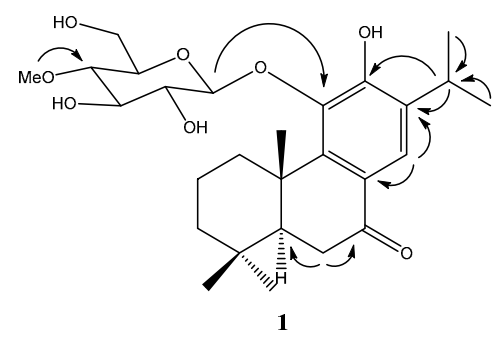

Figure 2. Key HMBC (from H to C) correlations for compound $\mathbf{1 .}$

Table 1. ${ }^{1} \mathrm{H}-\mathrm{NMR}$ (methanol- $\left.d_{4}, 600 \mathrm{MHz}\right),{ }^{13} \mathrm{C}-\mathrm{NMR}$ (methanol- $d_{4}, 150 \mathrm{MHz}$ ) data of compound 1 ( $\delta$ in ppm, $J$ in $\mathrm{Hz}$ ).

\begin{tabular}{|c|c|c|c|c|c|c|c|}
\hline No. & $\delta_{\mathrm{H}} J(\mathrm{~Hz})$ & $\delta_{C}$ & НМBC & No. & $\delta_{\mathrm{H}} J(\mathrm{~Hz})$ & $\delta_{C}$ & HМBC \\
\hline 1 & $\begin{array}{c}3.47(1 \mathrm{H}, \mathrm{d}, J=13.5 \mathrm{~Hz}) \\
1.40(1 \mathrm{H}, \mathrm{m})\end{array}$ & 38.3 & $\begin{array}{l}\text { C- } 2, C-3, \\
\text { C-5 }\end{array}$ & 15 & $3.27(1 \mathrm{H}, \mathrm{m})$ & 28.2 & $\begin{array}{l}\text { C-12, C-13, } \\
\text { C-14, C-17 }\end{array}$ \\
\hline 2 & $\begin{array}{c}1.81(1 \mathrm{H}, \mathrm{dt}, J=3.7,16.6 \mathrm{~Hz}) \\
1.56(1 \mathrm{H}, \mathrm{m})\end{array}$ & 20.1 & $C-3, C-5$ & 16 & $1.20(3 \mathrm{H}, \mathrm{d}, J=6.9 \mathrm{~Hz})$ & 22.8 & C-15, C-17 \\
\hline 3 & $\begin{array}{c}1.48(1 \mathrm{H}, \mathrm{d}, J=15.8 \mathrm{~Hz}) \\
1.29(1 \mathrm{H}, \mathrm{dd}, J=4.6,16.1 \mathrm{~Hz})\end{array}$ & 42.4 & $\begin{array}{l}\text { C- } 2, C-4, \\
\text { C-5 }\end{array}$ & 17 & $1.23(3 \mathrm{H}, \mathrm{d}, J=6.9 \mathrm{~Hz})$ & 22.5 & C-15,C-16 \\
\hline 4 & & 34.5 & & 18 & $0.93(3 \mathrm{H}, \mathrm{s})$ & 33.5 & $\begin{array}{l}\text { C-3, C- } 4, \\
\text { C-5, C-19 }\end{array}$ \\
\hline 5 & $1.76(1 \mathrm{H}, \mathrm{dd}, J=3.9,13.9 \mathrm{~Hz})$ & 51.7 & $\begin{array}{l}\text { C-3, C-4, } \\
\text { C-6, C-7 }\end{array}$ & 19 & $1.02(3 \mathrm{H}, \mathrm{s})$ & 22.0 & $\begin{array}{l}\text { C-3, C- } 4, \\
\text { C-5, C-18 }\end{array}$ \\
\hline 6 & $\begin{array}{l}2.61(1 \mathrm{H}, \mathrm{m}) \\
2.58(1 \mathrm{H}, \mathrm{m})\end{array}$ & 36.3 & $\begin{array}{l}\text { C- } 5, C-7, \\
\text { C- } 8\end{array}$ & 20 & $1.57(3 \mathrm{H}, \mathrm{s})$ & 20.5 & $\begin{array}{l}\text { C-1, C-3, } \\
\text { C-5, C-9 }\end{array}$ \\
\hline 7 & & 201.7 & & $1^{\prime}$ & $4.54(1 \mathrm{H}, \mathrm{d}, J=7.7 \mathrm{~Hz})$ & 106.8 & $C-2^{\prime}, C-11$ \\
\hline 8 & & 124.8 & & $2^{\prime}$ & $3.54(1 \mathrm{H}, \mathrm{m})$ & 75.9 & $C-1^{\prime}, C-3^{\prime}$ \\
\hline 9 & & 148.5 & & $3^{\prime}$ & $3.55(1 \mathrm{H}, \mathrm{m})$ & 78.1 & $\mathrm{C}-2^{\prime}, \mathrm{C}-4^{\prime}$ \\
\hline 10 & & 40.4 & & $4^{\prime}$ & $3.22(1 \mathrm{H}, \mathrm{m})$ & 80.3 & $C-3^{\prime}, C-5^{\prime}$ \\
\hline 11 & & 143.8 & & $5^{\prime}$ & $3.22(1 \mathrm{H}, \mathrm{m})$ & 77.9 & $C-4^{\prime}, C-6^{\prime}$ \\
\hline 12 & & 154.6 & & $6^{\prime}$ & $\begin{array}{l}3.81(1 \mathrm{H}, \mathrm{d}, J=11.3 \mathrm{~Hz}) \\
3.71(1 \mathrm{H}, \mathrm{d}, J=11.3 \mathrm{~Hz})\end{array}$ & 61.9 & $C-4^{\prime}, C-5^{\prime}$ \\
\hline 13 & & 136.1 & & $4^{\prime}-\mathrm{OCH}_{3}$ & $3.58(3 \mathrm{H}, \mathrm{s})$ & 60.8 & C- $4^{\prime}$ \\
\hline 14 & $7.73(1 \mathrm{H}, \mathrm{s})$ & 123.7 & $\begin{array}{c}\text { C-7, C-9, } \\
\text { C-11, } \\
\text { C-12 }\end{array}$ & & & & \\
\hline
\end{tabular}

By comparison of the spectroscopic and optical rotation values with the literature data, the 13 known compounds were identified as cyrtophyllones B (2) [10], uncinatone (3) [10], villosin B (4) [11], villosin C (5) [11], 3-O- $\alpha$-L-arabinopyranosyl olean-12-en-28-oic acid (6) [12], 3-O-[ $\beta$-D-glucopyranosyl 
(1-3)- $\alpha$-L-arabinopyranosyl)] hederagenin (7) [13], 3-O-[ $\beta$-D-glucopyranosyl(1-4)- $\alpha$-L-arabinopyranosyl)] olean-12-en-28-oic acid (8) [14], ciwujianoside A1 (9) [15], ciwujianoside C3 (10) [16], 2 $\alpha, 3 \alpha, 23$ trihydroxyoleane-12-en-28-oic acid (11) [17], (-)-syringaresinol (12) [18], medioresinol (13) [19], (2R,6R)-2-(4-hydroxy-3-methoxyphenyl)-6- (3,4,5-trimethoxypheny-1)-3,7-dioxabicyclo[3,3,0]octane (14) [20].

All isolated compounds were analyzed with respect to their inhibitory effects on PTP1B enzyme activity. Compounds 2, 3, 6, 8 and 11 significantly inhibited PTP1B activity, with IC $_{50}$ values ranging from $4.08 \pm 1.09$ to $21.80 \pm 4.74 \mu \mathrm{M}$ (Table 2). Compound 6 had an especially strong inhibitory effect on PTP1B enzyme, with an $\mathrm{IC}_{50}$ value of $4.08 \pm 1.09 \mu \mathrm{M}$, which was similar to that of the positive control (ursolic acid; $\mathrm{IC}_{50}$ value: $4.25 \pm 0.62 \mu \mathrm{M}$ ). As compounds 3 and 6 were potential PTP1B inhibitors, their inhibition mode was determined using a double reciprocal Lineweaver-Burk plot. Compounds 3 and $\mathbf{6}$ were found noncompetitive inhibitors because increasing the substrate concentrations resulted in a family of lines that intersect at a non-zero point on the negative $x$-axis (Figure 3). Compounds 2, 3, 6, 8 and 11 with PTP1B inhibition, were analyzed for their inhibitory effects against MCF7, MDA-MB-231 and MCF7/TAMR breast cancer cell lines. Four compounds 2, 3, 6 and 8, had strong cytotoxic activities against breast cancer lines, with $\mathrm{IC}_{50}$ values ranging from $0.84 \pm 0.17$ to $13.42 \pm 1.26 \mu \mathrm{M}$, compared with positive controls tamoxifen and 4-hydroxy tamoxifen $\left(\mathrm{IC}_{50}\right.$ values ranging from $2.11 \pm 0.39$ to $25.42 \pm 1.79 \mu \mathrm{M})$ (Table 2). Interestingly, while the effectiveness of the positive controls, tamoxifen and 4-OH tamoxifen, decreased by $1 / 3$ to 1/6 in the MCF7/TAMR cell line, compounds 6 and 8 showed similar cytotoxic effects between MCF7 and MCF7/TAMR cancer cells. These results suggest that compounds 6 and 8 could also be considered good inhibitors against tamoxifen-resistant cancer cell lines.

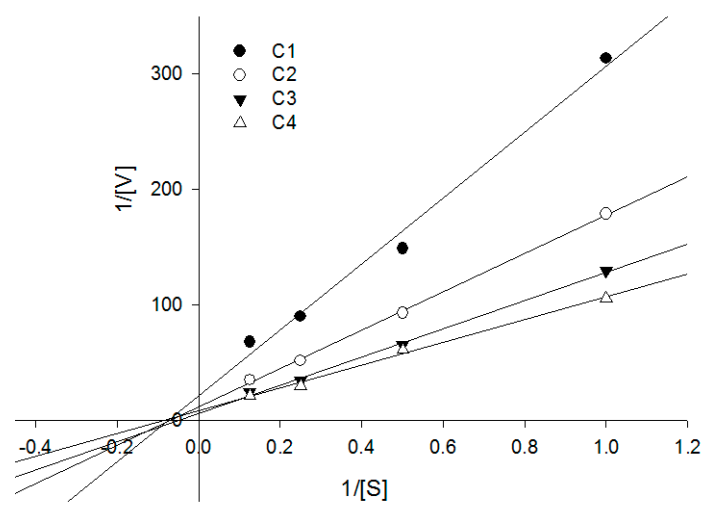

(A)

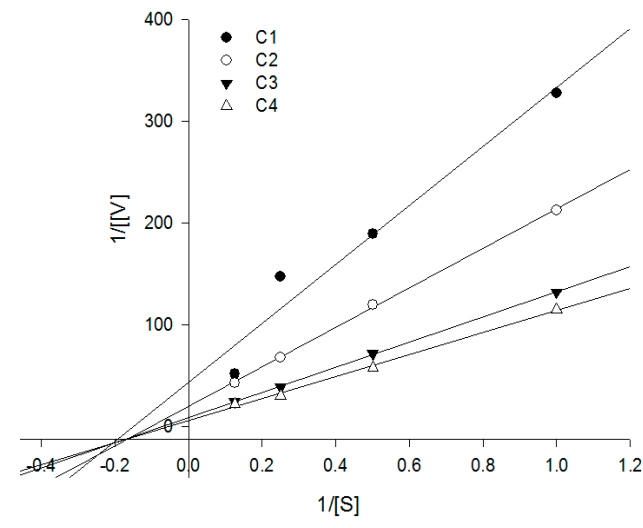

(B)

Figure 3. Graphical determination of the type of inhibition for compounds 3 and 6 . Lineweaver-Burk plots for the inhibition of compounds 3 (A) and 6 (B) on the PTP1B enzyme using $p$ NPP assay. The conditions were as follows: $4 \mathrm{mM}$ substrate, $0.05-0.1 \mu \mathrm{g} / \mathrm{mL}$ of PTP1B enzyme, $50 \mathrm{mM}$ Tris buffer ( $\mathrm{pH} 7.5$ ), at room temperature. In the presence of different concentrations of compounds for lines from bottom to top: (A) for compound 3, 0, 1.0, 2.0, 5.0, and $10.0 \mu \mathrm{M}$; (B) for compound 6, 0, 1.0, 2.0,5.0, and $10.0 \mu \mathrm{M}$. The data were evaluated in three replicates at each substrate concentration.

Apoptosis is the process of ordered and programmed cell death that occurs in both physiological and pathological conditions. As a chemopreventive strategy, apoptosis is considered one of the most promising treatments against breast cancer. Oblimersen, an apoptosis inducer, is presently in clinical trials with docetaxel, adriamycin and cyclophosphamide. Their preclinical and clinical potential, in combinations with many cancer medicines, against solid tumors is being used to discover therapeutic agents that effectively induce apoptosis [21]. The ability to induce apoptosis by compound 6 was determined by flow cytometry assay, using the ApopNexin ${ }^{\mathrm{TM}}$ FITC kit (Millipore, Billerica, MA, USA) to perform double-staining with Annexin FITC and propidium iodide (PI) in MCF-7 cells (with DMSO 
as a control). In the control group, $87.90 \%$ of cancer cells are viable, as shown in the lower left field (low Annexin V and PI staining). However, significant changes in the cancer cell profiles were observed when $10 \mu \mathrm{M}$ of compound $\mathbf{6}$ was added. The percentage of apoptotic cancer cells after $10 \mu \mathrm{M}$ treatment of compound 6 was increased to $89.47 \%$, as shown in the higher right field (Figure 4). These results suggested that compound 6 increased cancer cell cytotoxicity mainly by inducing cell apoptosis.

Table 2. Inhibitory effects of compounds $2,3,6,8$ and 11 on PTP1B enzyme activity and their cytotoxic activities against breast cancer cell lines.

\begin{tabular}{|c|c|c|c|c|}
\hline \multirow{2}{*}{ Compounds } & \multirow{2}{*}{$\operatorname{PTP1B~}\left(\mathrm{IC}_{50}, \mu \mathrm{M}\right)^{\mathrm{a}}$} & \multicolumn{3}{|c|}{ Cytotoxic Activities $\left(\mathrm{IC}_{50}, \mu \mathrm{M}\right)^{a}$} \\
\hline & & MCF7 & MDA-MB-231 & MCF7/TAMR \\
\hline 2 & $6.77 \pm 1.28$ & $7.91 \pm 0.39$ & $4.04 \pm 0.80$ & $13.42 \pm 1.26$ \\
\hline 3 & $5.41 \pm 0.68$ & $6.02 \pm 2.32$ & $5.14 \pm 1.55$ & $7.73 \pm 1.02$ \\
\hline 6 & $4.08 \pm 1.09$ & $1.11 \pm 0.04$ & $0.84 \pm 0.17$ & $1.39 \pm 0.18$ \\
\hline 8 & $21.80 \pm 4.74$ & $1.29 \pm 0.09$ & $1.55 \pm 0.21$ & $1.78 \pm 0.13$ \\
\hline 11 & $7.78 \pm 1.43$ & $>40$ & NT & NT \\
\hline Ursolic acid ${ }^{b}$ & $4.25 \pm 0.62$ & NT & NT & NT \\
\hline Tamoxifen ${ }^{b}$ & NT & $8.01 \pm 1.81$ & $10.09 \pm 1.51$ & $25.42 \pm 1.79$ \\
\hline 4-OH tamoxifen ${ }^{\mathrm{b}}$ & NT & $2.11 \pm 0.39$ & $3.10 \pm 0.31$ & $13.58 \pm 1.25$ \\
\hline
\end{tabular}

NT: not tested, ${ }^{a}$ Values are expressed as mean \pm SD of three replicates, ${ }^{b}$ Positive control.
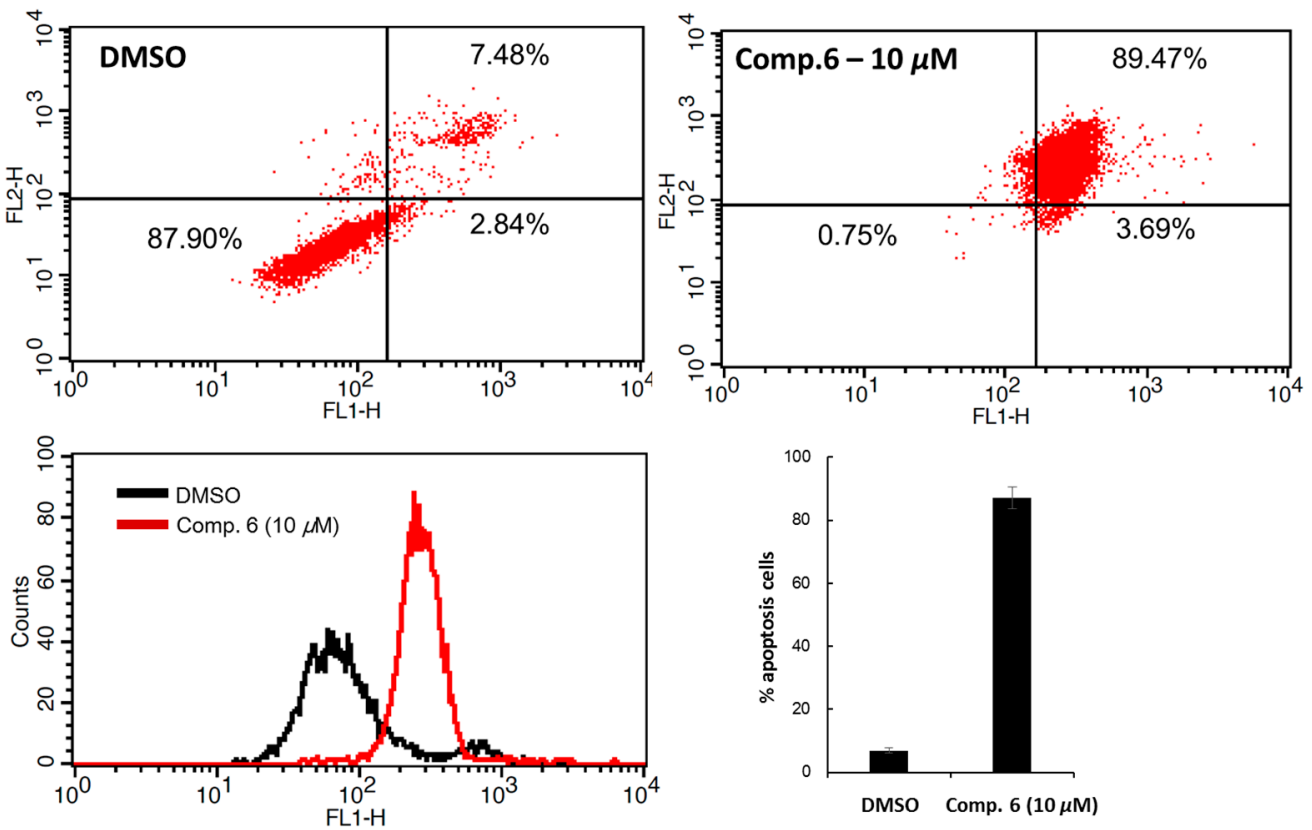

Figure 4. The inhibitory effect of compound 6 on MCF-7 cell apoptosis. The cells were treated with compound $6(10 \mu \mathrm{M})$ and incubated for $12 \mathrm{~h}$. Then, flow cytometric analysis was performed to detect cell apoptosis using the annexin conjugate ApopNexin ${ }^{\mathrm{TM}}$ FITC kit.

From a structural perspective, triterpene compounds $(6,8$ and 11$)$ with a COOH group at C-28 showed a significant enhancement of inhibitory activity against PTP1B enzyme. This result is consistent with previous studies that 28-COOH may be responsible for inhibiting the PTP1B enzyme [22]. In addition, compound 6 with one less glucopyranosyl moiety than that of compound 8, had stronger activity than compound 8 . This increased activity of compound 6 might be due to having one less glucopyranosyl, which is attached to C $-3^{\prime}$ of arabinopyranosyl. The presence of an alcohol group at C-23 may decrease the inhibitory effects on PTP1B. In agreement with a previous study, compound 8, which does not possess a hydroxyl group at C-23, had stronger inhibitory activity than compound 7. The same trend was also observed with diterpene compounds 3, 4 and 5 (data not shown). In a previous 
study, kaurane-type diterpenes without a hydroxy group at C-16 showed enhanced activity on PTP1B enzyme [23]. Among abietane type diterpenes, three tanshinones from root of Salvia miltiorrhiza have been reported to have PTP1B inhibitory effects. On the basis of our research, abietane diterpenes (2 and 3) from Akebia quinata were also found to have inhibitory effects on PTP1B enzyme.

\section{Experimental Section}

\subsection{General Information}

NMR spectra were recorded on a JEOL $600 \mathrm{MHz}$ spectrometer (JEOL, Tokyo, Japan) with TMS as the internal standard at the Seoul National University, Seoul, Korea. IR spectra $(\mathrm{KBr})$ were recorded on a Nicolet 6700 FT-IR (Thermo Fisher Scientific, Waltham, MA, USA). HRESIMS data were obtained with Agilent 1260 Infinity Q-TOF mass spectrometer (Agilent Technologies, Santa Clara, CA, USA). Optical rotation values were determined on a JASCO P-2000 polarimeter (JASCO, Tokyo, Japan). Silica gel (63-200 $\mu \mathrm{m}$ particle size, Merck, Darmstadt, Germany), and RP-C 18 (75 $\mu \mathrm{m}$ particle size, Nacalai Tesque, Kyoto, Japan) were used for column chromatography. TLC was carried out with silica gel $60 \mathrm{~F}_{254}$ and RP-C $\mathrm{C}_{18} \mathrm{~F}_{254}$ plates. HPLC was performed on a Gilson System with an Optima Pak $\mathrm{C}_{18}$ column (10 $\mu \mathrm{m}$ particle size, $10 \mathrm{~mm} \times 250 \mathrm{~mm}$; RS Tech, Seoul, Korea) and a UV detector. Analytical grade solvents were purchased from Fisher scientific (Pittsburgh, PA, USA).

\subsection{Extraction and Isolation}

The stems of A. quinata were collected in September in 2014 at Gangwon province, Korea. The dried stem $(10 \mathrm{~kg})$ of $A$. quinata were extracted with $90 \% \mathrm{MeOH}$ at room temperature for 1 week. The combined extract was concentrated under reduced pressure to yield a dried sample (400 g). This crude extract was then suspended in $\mathrm{H}_{2} \mathrm{O}(4 \mathrm{~L})$ and partitioned successively with $n$-hexane $(3 \times 4 \mathrm{~L})$, EtOAc $(3 \times 4 \mathrm{~L})$, and $n$-BuOH $(3 \times 4 \mathrm{~L})$. The partial EtOAc fraction $(47 \mathrm{~g})$ was chromatographed over a silica gel column $(5 \times 30 \mathrm{~cm} ; 63-200 \mu \mathrm{m}$ particle size $)$ and eluted with gradient mixtures of $n$-hexane/acetone $(10: 1 \rightarrow 1: 10$, each $2 \mathrm{~L})$ to afford six sub-fractions (Fr.1 to Fr.6). Fr.6 (4.3 g) was applied onto an RP-18 column $(4.5 \times 50 \mathrm{~cm} ; 75 \mu \mathrm{m}$ particle size $)$ using a $\mathrm{MeOH} / \mathrm{H}_{2} \mathrm{O}$ system (1:1.5 to 1:0) to yield eleven sub-fractions and compound 6 (21 mg). Fr.6-9

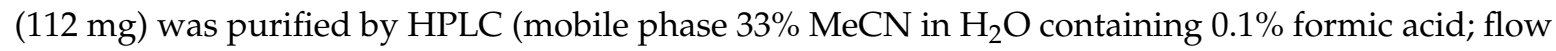
rate, $2 \mathrm{~mL} / \mathrm{min}$; UV detection at 205 and $254 \mathrm{~nm}$ ) using an isocratic solvent system to give compound 7 (5.6 mg; $\left.t_{\mathrm{R}}=30.0 \mathrm{~min}\right)$. Fr.6-10 (150 mg) was further purified using HPLC (mobile phase $\mathrm{MeCN} / \mathrm{H}_{2} \mathrm{O}$ containing $0.1 \%$ formic acid; 0-8 $\min$ : $10 \%-35 \% \mathrm{MeCN}, 8-12 \mathrm{~min}$ : 35\%-39\% MeCN, $12-24 \mathrm{~min}$ : 39\% MeCN, 25-34 min: 100\% MeCN, 35-39 min: 10\% MeCN; flow rate, $2 \mathrm{~mL} / \mathrm{min}$; UV detection at 205 and $254 \mathrm{~nm})$ to afford compound $8\left(10 \mathrm{mg} ; t_{\mathrm{R}}=23.2 \mathrm{~min}\right)$. Fr.6-11 $(185 \mathrm{mg})$ was also purified by HPLC (mobile phase $33 \% \mathrm{MeCN}$ in $\mathrm{H}_{2} \mathrm{O}$ containing $0.1 \%$ formic acid; 0-10 min: 60\% $\mathrm{MeOH}, 10-15 \mathrm{~min}$ : 60\%-75\% MeOH, $15-45$ min: 75\%-81\% MeOH, $45-55 \min : 100 \% \mathrm{MeOH}, 55-57 \mathrm{~min}$ : 60\% MeOH; flow rate, $2 \mathrm{~mL} / \mathrm{min}$; UV detection at 205 and $254 \mathrm{~nm}$ ) to yield compound 5 (5.0 mg; $\left.t_{\mathrm{R}}=38.3 \mathrm{~min}\right)$. Fr.5 ( $5 \mathrm{~g})$ was chromatographed on an RP-18 column $(4.5 \times 55 \mathrm{~cm}$; $75 \mu \mathrm{m}$ particle size $)$ and was eluted with a gradient $\mathrm{MeOH} / \mathrm{H}_{2} \mathrm{O}$ system (1:1 to 1:0) to afford 5 sub-fractions. Further purification of subfraction Fr.5-2 (102 mg) by HPLC, using an isocratic solvent system of $20 \% \mathrm{MeCN}$ in $\mathrm{H}_{2} \mathrm{O}$ containing $0.1 \%$ formic acid resulted in isolation of compound 2 ( $\left.3.3 \mathrm{mg} ; t_{\mathrm{R}}=20.5 \mathrm{~min}\right)$. Fr.4 (8 g) was subjected to an RP-18 column $(4.5 \times 40 \mathrm{~cm} ; 75 \mu \mathrm{m}$ particle size $)$ using $\mathrm{MeOH} / \mathrm{H}_{2} \mathrm{O}$ system $(1: 1$ to $1: 0)$ to give 10 subfractions. Fr.4-10 (90 mg) was further purified by HPLC with an isocratic solvent system of $89 \% \mathrm{MeOH}$ in $\mathrm{H}_{2} \mathrm{O}$ containing $0.1 \%$ formic acid to give compound $4\left(13 \mathrm{mg}\right.$; $\left.t_{\mathrm{R}}=30.7 \mathrm{~min}\right)$. Fr.3 $(10 \mathrm{~g})$ was fractionated into twelve subfractions (Fr.3-1 to Fr.3-12) and yielded compound 3 (32 mg) by RP-18 column $\left(4.5 \times 50 \mathrm{~cm} ; 75 \mu \mathrm{m}\right.$ particle size) eluted with $\mathrm{MeOH} / \mathrm{H}_{2} \mathrm{O}$ (1:1 to 1:0). Fr.3-3 (32 mg) was purified by HPLC (mobile phase $\mathrm{MeOH} / \mathrm{H}_{2} \mathrm{O}$ containing $0.1 \%$ formic acid; $0-7 \mathrm{~min}: 20 \%$ $\mathrm{MeOH}, 7-43 \mathrm{~min}: 28 \%-30 \% \mathrm{MeOH}, 43-46 \mathrm{~min}: 30 \%-34 \% \mathrm{MeOH}$, $46-55 \mathrm{~min}$ : 34\% MeOH, 55-64 min: $100 \% \mathrm{MeOH}, 64-67 \mathrm{~min}: 20 \% \mathrm{MeOH}$; flow rate, $2 \mathrm{~mL} / \mathrm{min}$; UV detection at 205 and $254 \mathrm{~nm}$ ) to give 
compounds $13\left(3.0 \mathrm{mg} ; t_{\mathrm{R}}=45.1 \mathrm{~min}\right)$ and $14\left(2.0 \mathrm{mg} ; t_{\mathrm{R}}=50.1 \mathrm{~min}\right)$. Fr.3-11 $(180 \mathrm{mg})$ was purified by HPLC, using an isocratic solvent system of $53 \% \mathrm{MeCN}$ in $\mathrm{H}_{2} \mathrm{O}$ containing $0.1 \%$ formic acid to give compounds $1\left(3.8 \mathrm{mg} ; t_{\mathrm{R}}=22.1 \mathrm{~min}\right), \mathbf{9}\left(2.5 \mathrm{mg} ; t_{\mathrm{R}}=33.7 \mathrm{~min}\right), \mathbf{1 0}\left(7.8 \mathrm{mg} ; t_{\mathrm{R}}=52.5 \mathrm{~min}\right)$ and $\mathbf{1 1}(5.6 \mathrm{mg}$; $\left.t_{\mathrm{R}}=28.8 \mathrm{~min}\right)$. Fr 3-12 (105 mg) was purified by HPLC using an isocratic solvent system of $52 \% \mathrm{MeCN}$ in $\mathrm{H}_{2} \mathrm{O}$ containing $0.1 \%$ formic acid to give compound $12\left(4.5 \mathrm{mg} ; t_{\mathrm{R}}=39.7 \mathrm{~min}\right)$.

Compound 1: Yellowish gum, $[\alpha]_{\mathrm{D}}^{20}+26.7(c 0.1, \mathrm{MeOH}) \mathrm{UV}(\mathrm{MeOH}) \lambda_{\max }(\log \varepsilon): 212(2.72), 232(2.36)$, 288 (2.72) nm; IR (KBr) vmax 3287, 2958, 2928, 2868, 1666, 1593, 1315, 1071, $1022 \mathrm{~cm}^{-1}$; HRESIMS positive-charged $m / z 493.2782[\mathrm{M}+\mathrm{H}]^{+}$(calcd. for $\mathrm{C}_{27} \mathrm{H}_{41} \mathrm{O}_{8}$, 493.2796) (Figure S8). ${ }^{1} \mathrm{H}-\mathrm{NMR}$ (methanol- $d_{4}, 600 \mathrm{MHz}$ ) and ${ }^{13} \mathrm{C}-\mathrm{NMR}$ (methanol- $d_{4}, 600 \mathrm{MHz}$ ) see Table 1.

Compound 2: Colorless needles, UV (MeOH), $\lambda_{\max } 212,227,290 \mathrm{~nm} ;{ }^{1} \mathrm{H}-\mathrm{NMR}$ (chloroform- $d, 600 \mathrm{MHz}$ ) ঠ: $7.45(1 \mathrm{H}, \mathrm{s}, \mathrm{H}-14), 4.00(1 \mathrm{H}, \mathrm{m}, \mathrm{H}-16 \mathrm{a}), 3.76(1 \mathrm{H}, \mathrm{m}, \mathrm{H}-16 \mathrm{~b}), 3.26(1 \mathrm{H}, \mathrm{d}, 13.5 \mathrm{~Hz}, \mathrm{H}-1 \mathrm{a}), 1.45(1 \mathrm{H}, \mathrm{m}$, H-1b), 3.14 (1H, m, H-15), 1.35 (3H, s, H-20), 1.33 (3H, d, 6.0 Hz, H-17), 0.93 (3H, s, H-19), 0.90 (3H, s, H-18); ${ }^{13}$ C-NMR (chloroform-d, $\left.600 \mathrm{MHz}\right)$ 8: 37.9 (C-1), 19.0 (C-2), 41.3 (C-3), 33.5 (C-4), 50.9 (C-5), 35.6 (C-6), 199.4 (C-7), 125.3 (C-8), 138.6 (C-9), 40.1 (C-10), 143.3 (C-11), 147.4 (C-12), 128.5 (C-13), 119.1 (C-14), 36.2 (C-15), 69.5 (C-16), 15.2 (C-17), 33.2 (C-18), 21.5 (C-19), 18.0 (C-20).

Compound 3: Yellow power, UV (MeOH), $\lambda_{\max } 225,283,304 \mathrm{~nm} ;{ }^{1} \mathrm{H}-\mathrm{NMR}$ (methanol- $d_{4}, 600 \mathrm{MHz}$ ) ઈ: $6.16(1 \mathrm{H}, \mathrm{s}, \mathrm{H}-6), 5.13(1 \mathrm{H}, \mathrm{ddq}, 7.9,6.9,6.4 \mathrm{~Hz}, \mathrm{H}-16), 3.43(1 \mathrm{H}, \mathrm{dd}, 13.2,5.3 \mathrm{~Hz}, \mathrm{H}-15 \mathrm{a}), 3.35(1 \mathrm{H}$, m, H-1a), 2.82 (1H, dd, 15.2, 7.3 Hz, H-15b), 1.93 (3H, s, H-18), 1.90 (3H, s, H-19), 1.51 (3H, s, H-20), $1.50(3 \mathrm{H}, \mathrm{d}, 6.4 \mathrm{~Hz}, \mathrm{H}-17) ;{ }^{13} \mathrm{C}-\mathrm{NMR}$ (methanol- $\left.d_{4}, 600 \mathrm{MHz}\right) \delta: 30.3$ (C-1), 31.2 (C-2), 142.4 (C-3), 126.3 (C-4), 168.0 (C-5), 118.8 (C-6), 191.6 (C-7), 109.9 (C-8), 138.5 (C-9), 41.2 (C-10), 133.5 (C-11), 154.7 (C-12), 112.6 (C-13), 156.8 (C-14), 34.9 (C-15), 83.6 (C-16), 22.3 (C-17), 20.6 (C-18), 15.1 (C-19), 22.2 (C-20).

Compound 4: Yellow power, UV (MeOH), $\lambda_{\max } 225,296 \mathrm{~nm} ;{ }^{1} \mathrm{H}-\mathrm{NMR}$ (methanol- $\left.d_{4}, 600 \mathrm{MHz}\right) \delta: 5.03$ (1H, m, H-16), 3.79 (1H, dd, 12.9, 4.3 Hz, H-17a), 3.69 (1H, dd, 12.1, $6.0 \mathrm{~Hz}, \mathrm{H}-17 \mathrm{~b}), 3.48$ (1H, d, 13.4 Hz, H-1a), 3.20 (1H, d, 15.4, 9.5 Hz, H-15a), 2.97 (1H, dd, 15.4, 7.0 Hz, H-15b), 1.34 (1H, m, H-1b), 1.38 (3H, s, H-20), 0.99 (3H, s, 19), 0.96 (3H, s, H-18); ${ }^{13} \mathrm{C}-\mathrm{NMR}$ (methanol- $\left.d_{4}, 600 \mathrm{MHz}\right) \delta: 37.6$ (C-1), 20.1 (C-2), 42.4 (C-3), 34.4 (C-4), 51.7 (C-5), 36.3 (C-6), 206.3 (C-7), 111.4 (C-8), 142.3 (C-9), 42.1 (C-10), 133.4 (C-11), 158.7 (C-12), 112.0 (C-13), 156.4 (C-14), 29.3 (C-15), 87.3 (C-16), 64.9 (C-17), 33.7 (C-18), 22.0 (C-19), 18.0 (C-20).

Compound 5: Yellow power, UV (MeOH), $\lambda_{\max } 225,267 \mathrm{~nm} ;{ }^{1} \mathrm{H}-\mathrm{NMR}$ (chloroform- $d, 600 \mathrm{MHz}$ ) $\delta: 5.13$ (1H, m, H-16), $3.93(1 \mathrm{H}, \mathrm{dd}, 12.2,2.6 \mathrm{~Hz}, \mathrm{H}-17 \mathrm{a}), 3.83(1 \mathrm{H}, \mathrm{dd}, 12.1,6.7 \mathrm{~Hz}, \mathrm{H}-17 \mathrm{~b}), 3.34$ (1H, dd, 15.4, $9.5 \mathrm{~Hz}, \mathrm{H}-15 \mathrm{a}), 3.05$ (1H, dd, 15.3, $7.3 \mathrm{~Hz}, \mathrm{H}-15 \mathrm{~b}), 3.06$ (1H, m, H-1a), 1.13 (1H, m, H-1b), 1.65 (3H, s, H-20), 1.44 (3H, s, H-19), 1.43 (3H, s, H-18); ${ }^{13} \mathrm{C}-\mathrm{NMR}$ (chloroform-d, $600 \mathrm{MHz}$ ) 8: 28.9 (C-1), 17.8 (C-2), 42.0 (C-3), 36.4 (C-4), 144.5 (C-5), 141.6 (C-6), 183.0 (C-7), 107.2 (C-8), 140.2 (C-9), 36.7 (C-10), 130.9 (C-11), 153.3 (C-12), 110.5 (C-13), 154.4 (C-14), 30.0 (C-15), 86.5 (C-16), 64.9 (C-17), 28.1 (C-18), $27.2(\mathrm{C}-19), 27.5$ (C-20).

Compound 6: White powder, UV (MeOH), $\lambda_{\max } 205 \mathrm{~nm} ;{ }^{1} \mathrm{H}-\mathrm{NMR}$ (pyridine- $\left.d_{5}, 300 \mathrm{MHz}\right) \delta: 5.48(1 \mathrm{H}$, t-like, H-12), 4.78 (1H, d, 7.1 Hz, Ara-H-1), 1.29 (3H, s, H-27), 1.27 (3H, s, H-23), 1.00 (6H, s, H3-24,30), $0.99(6 \mathrm{H}, \mathrm{s}, \mathrm{H} 3-26,29), 0.85$ (3H, s, H-25); ${ }^{13} \mathrm{C}-\mathrm{NMR}$ (pyridine- $\left.d_{5}, 300 \mathrm{MHz}\right) \delta: 38.8$ (C-1), 26.6 (C-2), 88.4 (C-3), 39.6 (C-4), 55.9 (C-5), 18.5 (C-6), 33.2 (C-7), 39.7 (C-8), 48.0 (C-9), 37.0 (C-10), 23.7 (C-11), 122.6 (C-12), 144.8 (C-13), 42.2 (C-14), 28.3 (C-15), 23.7 (C-16), 46.7 (C-17), 41.9 (C-18), 46.5 (C-19), 30.9 (C-20), 34.2 (C-21), 33.3 (C-22), 28.2 (C-23), 16.9 (C-24), 15.5 (C-25), 17.4 (C-26), 26.2 (C-27), 180.2 (C-28), 33.2 (C-29), 23.8 (C-30), 107.5 (Ara-1), 72.9 (Ara-2), 74.6 (Ara-3), 69.5 (Ara-4), 66.8 (Ara-5).

Compound 7: White power, UV (MeOH), $\lambda_{\max } 205 \mathrm{~nm} ;{ }^{1} \mathrm{H}-\mathrm{NMR}$ (pyridine- $\left.d_{5}, 600 \mathrm{MHz}\right) \delta: 5.48(1 \mathrm{H}$, t-like, H-12), 5.35 (1H, d, 7.6 Hz, Glc-H-1), 5.00 (1H, d, 7.4 Hz, Ara-H-1), 1.26 (3H, s, H-27), $1.02(3 \mathrm{H}, \mathrm{s}$, 
H-25), 0.99 (3H, s, H-30), 0.93 (6H, s, H3-26,29), 0.92 (3H, s, H-24); ${ }^{13} \mathrm{C}-\mathrm{NMR}$ (pyridine- $d_{5}, 600 \mathrm{MHz}$ )

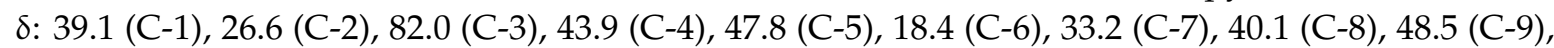
37.2 (C-10), 24.2 (C-11), 122.9 (C-12), 145.1 (C-13), 42.5 (C-14), 28.7 (C-15), 24.0 (C-16), 47.0 (C-17), 42.3 (C-18), 46.7 (C-19), 31.3 (C-20), 34.5 (C-21), 33.6 (C-22), 64.4 (C-23), 14.0 (C-24), 16.4 (C-25), 17.8 (C-26), 26.5 (C-27), 180.6 (C-28), 33.5 (C-29), 24.1 (C-30), 106.8 (Ara-1), 72.4 (Ara-2), 84.6 (Ara-3), 69.7 (Ara-4), 67.8 (Ara-5), 107.0 (Glc-1), 76.1 (Glc-2), 78.8 (Glc-3), 71.9 (Glc-4), 79.1 (Glc-5), 63.0 (Glc-6)

Compound 8: White powder, UV (MeOH), $\lambda_{\max } 205 \mathrm{~nm} ;{ }^{1} \mathrm{H}-\mathrm{NMR}$ (methanol- $\left.d_{4}, 400 \mathrm{MHz}\right) \delta: 5.24(1 \mathrm{H}$, t-like, H-12), $4.59(1 \mathrm{H}, \mathrm{d}, 7.8 \mathrm{~Hz}$, Glc-H-1), $4.50(1 \mathrm{H}, \mathrm{d}, 6.0 \mathrm{~Hz}$, Ara-H-1), $2.86(1 \mathrm{H}, \mathrm{dd}, 13.5,3.8 \mathrm{~Hz}$, H-18), 1.16 (3H, s, H-27), 1.06 (3H, s, H-23), 0.96 (3H, s, H-24), 0.94 (3H, s, H-30), 0.91 (3H, s, H-26), 0.85 (3H, s, H-29), 0.82 (3H, s, H-25); ${ }^{13} \mathrm{C}-\mathrm{NMR}$ (methanol- $\left.d_{4}, 400 \mathrm{MHz}\right) \delta: 40.4$ (C-1), 27.0 (C-2), 91.2 (C-3), 40.4 (C-4), 56.9 (C-5), 19.4 (C-6), 33.8 (C-7), 40.6 (C-8), 47.6 (C-9), 37.9 (C-10), 24.0 (C-11), 123.6 (C-12), 145.2 (C-13), 42.9 (C-14), 28.9 (C-15), 23.9 (C-16), 47.3 (C-17), 42.7 (C-18), 47.3 (C-19), 31.6 (C-20), 34.9 (C-21), 34.0 (C-22), 28.6 (C-23), 16.9 (C-24), 15.9 (C-25), 17.7 (C-26), 26.4, (C-27), 181.8 (C-28), 33.6 (C-29), 24.5 (C-30), 104.8 (Ara-1), 68.9 (Ara-2), 73.7 (Ara-3), 79.1 (Ara-4), 65.2 (Ara-5), 105.4 (Glc-1), 75.9 (Glc-2), 78.2 (Glc-3), 71.7 (Glc-4), 77.9 (Glc-5), 62.9 (Glc-6).

Compound 9: White powder, UV (MeOH), $\lambda_{\max } 205 \mathrm{~nm} ;{ }^{1} \mathrm{H}-\mathrm{NMR}$ (methanol- $\left.d_{4}, 600 \mathrm{MHz}\right) \delta: 5.34$ $\left(1 \mathrm{H}, \mathrm{d}, 8.0 \mathrm{~Hz}, \mathrm{Glc}^{\prime}-\mathrm{H}-1\right), 5.26$ (1H, t-like, H-12), 4.89 (1H, s, Rha-H-1), 4.59 (1H, d, 7.7 Hz, Glc'-H-1), $4.52(1 \mathrm{H}, \mathrm{d}, 6.0 \mathrm{~Hz}$, Ara-H-1), $4.41(1 \mathrm{H}, \mathrm{d}, 7.9 \mathrm{~Hz}, \mathrm{Glc}-\mathrm{H}-1), 1.27(3 \mathrm{H}, \mathrm{d}, 6.2 \mathrm{~Hz}$, Rha-H-6), 1.15 (3H, s, H-27), 1.05 (3H, s, H-23), 0.96 (3H, s, H-30), 0.95 (3H, s, H-24), 0.91 (3H, s, H-26), 0.85 (3H, s, H-29), 0.79 (3H, s, H-25); ${ }^{13} \mathrm{C}-\mathrm{NMR}$ (methanol- $\left.d_{4}, 600 \mathrm{MHz}\right) \delta$ : 39.8 (C-1), 27.0 (C-2), 91.2 (C-3), 40.4 (C-4), 56.9 (C-5), 19.4 (C-6), 33.9 (C-7), 40.7 (C-8), 49.0 (C-9), 37.9 (C-10), 24.1 (C-11), 123.8 (C-12), 144.9 (C-13), 42.9 (C-14), 28.9 (C-15), 24.0 (C-16), 48.1 (C-17), 42.5 (C-18), 47.3 (C-19), 31.6 (C-20), 34.9 (C-21), 33.5 (C-22), 28.6 (C-23), 16.9 (C-24), 16.1 (C-25), 17.8 (C-26), 26.3 (C-27), 178.1 (C-28), 33.3 (C-29), 24.6 (C-30), 105.4 (Ara-1), 79.6 (Ara-2), 72.2 (Ara-3), 68.9 (Ara-4), 104.2 (Glc-1), 75.9 (Glc-2), 78.1 (Glc-3), 71.7 (Glc-4), 78.0 (Glc-5), 62.9 (Glc-6), 95.8 (Glc'-1), 73.8 (Glc'-2), 78.2 (Glc'-3), 70.9 (Glc'-4), 78.2 (Glc'-5), 69.4 (Glc'-6),

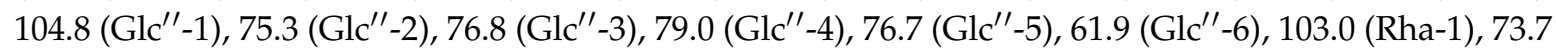
(Rha-2), 72.4 (Rha-3), 73.9 (Rha-4), 70.7 (Rha-5), 17.9 (Rha-6).

Compound 10: White powder, UV (MeOH), $\lambda_{\max } 205 \mathrm{~nm} ;{ }^{1} \mathrm{H}-\mathrm{NMR}$ (pyridine- $\left.d_{5}, 600 \mathrm{MHz}\right) \delta: 6.28$ $(1 \mathrm{H}, \mathrm{d}, 8.4 \mathrm{~Hz}$, Glc-H-1), 5.90 (1H, s, Rha-H-1), 5.42 (1H, s, H-12), 5.02 (1H, d, 7.8 Hz, Glc'-H-1), 4.79 $(1 \mathrm{H}, \mathrm{d}, 6.6 \mathrm{~Hz}$, Ara-H-1), 1.72 (3H, d, $6.2 \mathrm{~Hz}$, Rha-H-6), 1.25 (3H, s, H-27), 1.24 (3H, s, H-23), 1.15 (3H, s, H-30), 1.00 (3H, s, H-24), 0.90 (9H, s, H3-25,26,29); ${ }^{13} \mathrm{C}-\mathrm{NMR}$ (pyridine- $d_{5}, 600 \mathrm{MHz}$ ) $\delta: 39.1$ (C-1), 27.0 (C-2), 89.0 (C-3), 39.9 (C-4), 56.2 (C-5), 18.9 (C-6), 33.5 (C-7), 40.0 (C-8), 48.4 (C-9), 37.4 (C-10), 24.2 (C-11), 123.2 (C-12), 144.5 (C-13), 42.5 (C-14), 28.6 (C-15), 24.0 (C-16), 47.4 (C-17), 42.0 (C-18), 46.5 (C-19), 31.1 (C-20), 34.3 (C-21), 32.9 (C-22), 28.6 (C-23), 17.3 (C-24), 15.9 (C-25), 17.9 (C-26), 26.4 (C-27), 176.9 (C-28), 33.4 (C-29), 24.0 (C-30), 108.0 (Ara-1), 74.2 (Ara-2), 75.1 (Ara-3), 70.0 (Ara-4), 67.3 (Ara-5), 96.0 (Glc-1), 74.4 (Glc-2), 79.1 (Glc-3), 71.2 (Glc-4), 78.4 (Glc-5), 69.5 (Glc-6), 105.3 (Glc'-1), 75.7 (Glc'-2), 76.9 (Glc'-3), 78.5 (Glc'-4), 77.6 (Glc'-5), 61.6 (Glc'-6), 103.1 (Rha-1), 72.9 (Rha-2), 73.2 (Rha-3), 73.3 (Rha-4), 70.7 (Rha-5), 18.9 (Rha-6).

Compound 11: White powder, UV (MeOH), $\lambda_{\max } 205 \mathrm{~nm} ;{ }^{1} \mathrm{H}-\mathrm{NMR}$ (DMSO- $\left.d_{6}, 300 \mathrm{MHz}\right) \delta: 5.16(1 \mathrm{H}$, t-like, H-12), $4.02(1 \mathrm{H}, \mathrm{m}, \mathrm{H}-2), 3.92(1 \mathrm{H}, \mathrm{m}, \mathrm{H}-3), 1.09(3 \mathrm{H}, \mathrm{s}, \mathrm{H}-27), 0.93(3 \mathrm{H}, \mathrm{s}, \mathrm{H}-25), 0.87$ (6H, s, H3-26,30), 0.85 (3H, s, H-29), 0.67 (3H, s, H-24); ${ }^{13} \mathrm{C}-\mathrm{NMR}$ (DMSO-d $\left.6,300 \mathrm{MHz}\right)$ 8: 43.1 (C-1), 66.3 (C-2), 74.3 (C-3), 42.3 (C-4), 49.6 (C-5), 19.0 (C-6), 33.8 (C-7), 40.1 (C-8), 48.3 (C-9), 38.6 (C-10), 24.2 (C-11), 122.5 (C-12), 144.9 (C-13), 42.1 (C-14), 28.3 (C-15), 24.0 (C-16), 46.7 (C-17), 42.1 (C-18), 45.2 (C-19), 31.0 (C-20), 34.3 (C-21), 34.1 (C-22), 23.9 (C-23), 65.3 (C-24), 17.6 (C-25), 17.6 (C-26), 26.2 (C-27), 180.4 (C-28), 33.3 (C-29), 23.9 (C-30). 
Compound 12: Colorless needle, UV (MeOH), $\lambda_{\max } 237,273 \mathrm{~nm} ;{ }^{1} \mathrm{H}-\mathrm{NMR}$ (methanol- $\left.d_{4}, 300 \mathrm{MHz}\right) \delta$ : $6.60\left(4 \mathrm{H}, \mathrm{s}, \mathrm{H}-2^{\prime}, 6^{\prime}, 2^{\prime \prime}, 6^{\prime \prime}\right), 4.70(2 \mathrm{H}, \mathrm{d}, \mathrm{H}-2,6), 4.20$ (2H, dd, 8.7, 6.9 Hz, H-4a,8a), 3.80 (2H, dd, 9.1, 3.6 $\mathrm{Hz}, \mathrm{H}-4 \mathrm{~b}, 8 \mathrm{~b}), 3.83$ (12H, s, OMe), 3.10 (2H, dd, 6.4, 4.6 Hz, H-1,5); ${ }^{13} \mathrm{C}-\mathrm{NMR}$ (methanol-d, $\left.300 \mathrm{MHz}\right) \delta$ : 54.3 (C-1), 86.0 (C-2), 71.8 (C-4), 54.3 (C-5), 86.0 (C-6), $71.8(\mathrm{C}-8), 132.0\left(\mathrm{C}-1^{\prime}\right), 102.7\left(\mathrm{C}-2^{\prime}\right), 147.1\left(\mathrm{C}-3^{\prime}\right)$, $134.3\left(\mathrm{C}-4^{\prime}\right), 147.1\left(\mathrm{C}-5^{\prime}\right), 102.7\left(\mathrm{C}-6^{\prime}\right), 132.0\left(\mathrm{C}-1^{\prime \prime}\right), 102.7\left(\mathrm{C}-2^{\prime \prime}\right), 147.1\left(\mathrm{C}-3^{\prime \prime}\right), 134.3\left(\mathrm{C}-4^{\prime \prime}\right), 147.1\left(\mathrm{C}-5^{\prime \prime}\right)$, $102.7\left(\mathrm{C}-6^{\prime \prime}\right)$.

Compound 13: Colorless needle, UV (MeOH), $\lambda_{\max } 231,280 \mathrm{~nm} ;{ }^{1} \mathrm{H}-\mathrm{NMR}$ (methanol- $d_{4}, 300 \mathrm{MHz}$ ) $\delta: 6.96\left(1 \mathrm{H}, \mathrm{d}, 1.5 \mathrm{~Hz}, \mathrm{H}-6^{\prime \prime}\right), 6.83\left(1 \mathrm{H}, \mathrm{dd}, 8.5,1.5 \mathrm{~Hz}, \mathrm{H}-2^{\prime \prime}\right), 6.78\left(1 \mathrm{H}, \mathrm{d}, 8.5 \mathrm{~Hz}, \mathrm{H}-3^{\prime \prime}\right), 6.66(2 \mathrm{H}, \mathrm{s}$, $\left.\mathrm{H}-2^{\prime}, 6^{\prime}\right), 4.72(2 \mathrm{H}, \mathrm{d}, 4.0 \mathrm{~Hz}, \mathrm{H}-2,6), 4.27$ (2H, m, H-4a,8a), 3.87 (3H, s, OMe-5'), $3.85\left(6 \mathrm{H}, \mathrm{s}, \mathrm{OMe}-3^{\prime}, 5^{\prime}\right)$, $3.82(2 \mathrm{H}, \mathrm{m}, \mathrm{H}-4 \mathrm{~b}, 8 \mathrm{~b}), 3.16(2 \mathrm{H}, \mathrm{m}, \mathrm{H}-1,5) ;{ }^{13} \mathrm{C}-\mathrm{NMR}$ (methanol- $\left.d_{4}, 300 \mathrm{MHz}\right) \delta: 55.2(\mathrm{C}-1), 87.5(\mathrm{C}-2)$, 72.5 (C-4), 55.4 (C-5), 87.4 (C-6), $72.6(\mathrm{C}-8), 133.0\left(\mathrm{C}-1^{\prime}\right), 104.3\left(\mathrm{C}-2^{\prime}\right), 149.2\left(\mathrm{C}-3^{\prime}\right), 136.0\left(\mathrm{C}-4^{\prime}\right), 149.2$ $\left(\mathrm{C}-5^{\prime}\right), 104.3\left(\mathrm{C}-6^{\prime}\right), 133.6\left(\mathrm{C}-1^{\prime \prime}\right), 120.0\left(\mathrm{C}-2^{\prime \prime}\right), 116.0\left(\mathrm{C}-3^{\prime \prime}\right), 147.2\left(\mathrm{C}-4^{\prime \prime}\right), 149.0\left(\mathrm{C}-5^{\prime \prime}\right), 111.0\left(\mathrm{C}-6^{\prime \prime}\right), 56.6$ $\left(\mathrm{OMe}-3^{\prime}, 5^{\prime}\right), 56.2\left(\mathrm{OMe}-5^{\prime \prime}\right)$.

Compound 14: Colorless needle, UV (MeOH), $\lambda_{\max } 210,280 \mathrm{~nm} ;{ }^{1} \mathrm{H}-\mathrm{NMR}$ (chloroform- $\left.d, 600 \mathrm{MHz}\right) \delta$ : $6.94\left(1 \mathrm{H}, \mathrm{s}, \mathrm{H}-2^{\prime}\right), 6.85\left(1 \mathrm{H}, \mathrm{d}, 8.0 \mathrm{~Hz}, \mathrm{H}-5^{\prime}\right), 6.73\left(1 \mathrm{H}, \mathrm{d}, 8.0, \mathrm{H}-6^{\prime}\right) ;{ }^{13} \mathrm{C}-\mathrm{NMR}$ (chloroform-d, $\left.600 \mathrm{MHz}\right)$

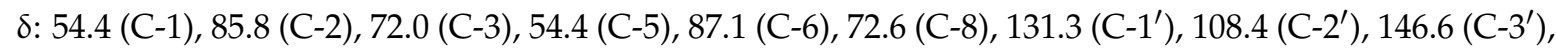
$144.9\left(\mathrm{C}-4^{\prime}\right), 114.2\left(\mathrm{C}-5^{\prime}\right), 118.8\left(\mathrm{C}-6^{\prime}\right), 137.6\left(\mathrm{C}-1^{\prime \prime}\right), 102.8\left(\mathrm{C}-2^{\prime \prime}\right), 153.5\left(\mathrm{C}-3^{\prime \prime}\right), 137.6\left(\mathrm{C}-4^{\prime \prime}\right), 153.5\left(\mathrm{C}-5^{\prime \prime}\right)$, $102.8\left(\mathrm{C}-6^{\prime \prime}\right) .60 .6,56.3,56.3,56.0(\mathrm{OMe})$.

\section{3. РТР1B Enzyme Assay}

PTP1B (human, recombinant) was purchased from BIOMOL International LP (USA) and the enzyme activity was measured using $p$-nitrophenyl phosphate ( $p$-NPP) (N1891, Sigma-Aldrich, St. Louis, MO, USA) as a substrate. In general, the assay was performed in 96-well plate containing $4 \mathrm{mM} p$-NPP and PTP1B (0.05-0.1 $\mu \mathrm{g}$ ) in enzyme buffer (50 mM citrate ( $\mathrm{pH} 6.0), 0.1 \mathrm{M} \mathrm{NaCl}, 1 \mathrm{mM}$ dithiothreitol (DTT), and $1 \mathrm{mM}$ EDTA) with or without test compounds. The assay was also carried out in the absence of PTP1B enzyme to exclude the effect of test compounds on the measurement absorbance. Following incubation at $37^{\circ} \mathrm{C}$ for $30 \mathrm{~min}$, the reaction was terminated with $10 \mathrm{M} \mathrm{NaOH}$ solution. The amount of produced $p$-nitrophenol was estimated by measuring the absorbance at 405 nm using an absorbance microplate reader (VersaMax ${ }^{\mathrm{TM}}$, Randor, PA, USA) [24]. For the enzyme kinetic determination, Lineweaver-Burk was used to determine the inhibition type of PTP1B. On the basis of Lineweaver-Burk double reciprocal plots, the PTP1B inhibition mode was obtained at various concentrations of p-NPP substrate $(1,2,4$, and $8 \mathrm{mM})$ without or with different test compound concentrations $(1,2,5$, and $10 \mu \mathrm{M})$. The $50 \%$ inhibition concentration $\left(\mathrm{IC}_{50}\right)$ and inhibition type were evaluated using Sigma Plot Statistical Analysis software (11.0 software, SPCC Inc., Chicago, IL, USA).

\subsection{Flow Cytometric Analysis of the Cell Apoptosis}

An ApopNexin ${ }^{\mathrm{TM}}$ FITC kit (Millipore) was used for the flow cytometric analysis. After MCF-7 cells were seeded on 6-well plates and incubated for $24 \mathrm{~h}$, the cells were treated with test compounds and incubated for $12 \mathrm{~h}$. The detached cells were collected and resuspended to wash the cells twice with PBS. The cells were suspended in cold binding buffer (10 mM Hepes/NaOH pH 7.4, $140 \mathrm{mM}$ $\mathrm{NaCl}, 2.5 \mathrm{mM} \mathrm{CaCl}_{2}$ ) at a concentration of $10^{6}$ cells $/ \mathrm{mL}$. Annexin conjugate ApopNexin ${ }^{\mathrm{TM}}$ FITC and propidium iodide (PI) were added to the cell suspension, which was incubated for 15 min in the dark. Then, the double-stained cells were immediately analyzed using a flow cytometer (BD bioscience, San Jose, CA, USA).

\section{Conclusions}

In this study, bioassay-guided fractionation of a methanol extract from Akebia quinata stems yielded one new diterpene glycoside (1) and 13 known compounds (2-14). Five compounds (2, 3, 6, 8 
and 11) exhibited potential inhibitory effects on PTP1B enzyme activity and cytotoxic effects on breast cancer cell lines. Using a flow-cytometry assay, compound 6 was further found to induce apoptosis in breast cancer cells. Therefore, these compounds may be developed as PTP1B inhibitors and anti-breast cancer agents.

Supplementary Materials: Spectral data $\left({ }^{1} \mathrm{H}-,{ }^{13} \mathrm{C}-\mathrm{NMR}, \mathrm{HSQC}\right.$ and HMBC) of compound 1 are available free of charge on the Internet at http:/ /www.mdpi.com/1420-3049/21/8/1091/s1.

Acknowledgments: This work was supported in part by grants from the Marine Biotechnology Program of the Ministry of Oceans and Fisheries (PJT200669) and the Korea Bioactive Natural Material Bank (NRF-2012M3A9B8021570) of the National Research Foundation of Korea (NRF), which is funded by the Korean government.

Author Contributions: T.O.C. and W.K.O. designed the experiments; J.P.A. and T.K.Q.H. performed the experiments; W.K.O. and J.K. analyzed the data; J.P.A. and W.K.O wrote the paper. All authors read and approved the final manuscript.

Conflicts of Interest: The authors declare no conflict of interest.

\section{References}

1. Vasudevan, D.; Jayalakshmy, P.S.; Kumar, S.; Mathew, S. Assessment of pathological response of breast carcinoma in modified radical mastectomy specimens after neoadjuvant chemotherapy. Int. J. Breast Cancer 2015, 2015, 536145-536152. [CrossRef] [PubMed]

2. Mariotto, A.B.; Yabroff, K.R.; Shao, Y.; Feuer, E.J.; Brown, M.L. Projections of the cost of cancer care in the United States: 2010-2020. J. Natl. Cancer Inst. 2011, 103, 117-128. [CrossRef] [PubMed]

3. Wiener, J.R.; Kerns, B.J.M.; Harvey, E.L.; Conaway, M.R.; Iglehart, J.D.; Berchuck, A.; Bast, R.C., Jr. Overexpression of the protein-tyrosine-phosphatase $1 \mathrm{~B}$ in human breast-cancer-association with P185(C-Erbb-2) protein expression. J. Natl. Cancer Inst. 1994, 86, 372-378. [CrossRef] [PubMed]

4. Blanquart, C.; Karouri, S.E.; Issad, T. Protein tyrosine phosphatase-1B and T-cell protein tyrosine phosphatase regulate IGF-2-induced MCF-7 cell migration. Biochem. Biophys. Res. Commun. 2010, 392, 83-88. [CrossRef] [PubMed]

5. Mohamed, B.A.; Benjamin, G.N. Protein-tyrosine phosphatase 1B is required for HER2/Neu-induced breast cancer. Cancer Res. 2007, 67, 2420-2424.

6. Gonzalez-Rodriguez, A.; Mas Gutierrez, J.A.; Sanz-Gonzalez, S.; Ros, M.; Burks, D.J.; Valverde, A.M. Inhibition of PTP1B restores IRS1-mediated hepatic insulin signaling in IRS2-deficient mice. Diabetes 2010, 59, 588-599. [CrossRef] [PubMed]

7. Ma, Y.M.; Tao, R.Y.; Liu, Q.; Li, J.; Tian, J.Y.; Zhang, X.L.; Xiao, Z.Y.; Ye, F. PTP1B inhibitor improves both insulin resistance and lipid abnormalities in vivo and in vitro. Mol. Cell. Biochem. 2011, 357, 65-72. [CrossRef] [PubMed]

8. Mimaki, Y.; Doi, S.; Kuroda, M.; Yokosuka, A. Triterpene glycosides from the stems of Akebia quinata. Chem. Pharm. Bull. 2007, 55, 1319-1324. [CrossRef] [PubMed]

9. Koo, H.J.; Sung, Y.Y.; Kim, H.K. Inhibitory effects of Akebia quinata ethanol extract on TNF- $\alpha$-mediated vascular inflammation in human aortic smooth muscle cells. Mol. Med. Rep. 2013, 7, 379-383. [PubMed]

10. Tian, X.; Min, Z.; Xie, N.; Lei, Y.; Tian, Z.; Zheng, Q.; Xu, R.; Tanaka, T.; Iinuma, M.; Mizuno, M. Abietane diterpenes from Clerodendron cyrtophyllum. Chem. Pharm. Bull. 1993, 41, 1415-1417. [CrossRef]

11. Ulubelen, A.; Topcu, G.; Olcal, S. Rearranged abietane diterpenes from Teucrium divaricatum Subsp. Villosum. Phytochemistry 1994, 37, 1371-1375. [CrossRef]

12. Morgan, A.M.A.; Kim, J.H.; Lee, H.W.; Lee, S.H.; Lim, C.; Jang, H.; Kim, Y.H. Phytochemical constituents from the aerial part of Ducrosia ismaelis Asch. Nat. Prod. Sci. 2015, 21, 6-13.

13. Martin, P.K.C.; Laurence, V.N. Synthesis of L-arabinopyranose containing hederagenin saponins. Tetrahedron 2005, 61, 4347-4362.

14. Grishkovets, V.I.; Sobolev, E.A.; Shashkov, A.S.; Chirva, V.Y. Triterpenoid glycosides of Fatsia japonica. II. Isolation and structure of glycosides from the leaves. Chem. Nat. Compd. 2000, 36, 501-505. [CrossRef]

15. Shao, C.J.; Kasai, R.; Xu, J.D.; Tanaka, O. Saponins from leaves of Acanthopanax senticosus Harms., ciwujia. II.: Structures of ciwujianosides $\mathrm{A}_{1}, \mathrm{~A}_{2}, \mathrm{~A}_{3}, \mathrm{~A}_{4}$ and $\mathrm{D}_{3}$. Chem. Pharm. Bull. 1989, 37, 42-45. [CrossRef] 
16. Shao, C.J.; Kasai, R.; Xu, J.D.; Tanaka, O. Saponins from leaves of Acanthopanax senticosus HARMS., ciwujia: Structures of ciwujianosides B, $C_{1}, C_{2}, C_{3}, C_{4}, D_{1}, D_{2}$ and E. Chem. Pharm. Bull. 1988, 36, 601-608. [CrossRef]

17. Ullah, F.; Hussain, H.; Hussain, J.; Bukhari, I.A.; Khan, M.T.; Choudhary, M.I.; Gilani, A.H.; Ahmad, V.U. Tyrosinase inhibitory pentacyclic triterpenes and analgesic and spasmolytic activities of methanol extracts of Rhododendron collettianum. Phytother. Res. 2007, 21, 1076-1081. [CrossRef] [PubMed]

18. Chen, B.N.; Yang, G.E.; Li, J.K.; Du, H.J.; Li, Q.S.; Zhang, Z.M. Cytotoxic constituents from Viscum coloratum. Chem. Nat. Compd. 2009, 45, 547-549. [CrossRef]

19. Tsukamoto, H.; Hisada, S.; Nishibe, S. Lignans from bark of Fraxinus mandshurica var. japonica and F. japonica. Chem. Pharm. Bull. 1984, 32, 4482-4489. [CrossRef]

20. Miyazawa, M.; Kasahara, H.; Kameoka, H. Phenolic lignans from flower buds of Magnolia fargesii. Phytochemistry 1992, 31, 3666-3668. [CrossRef]

21. Wu, J. Apoptosis and angiogenesis: Two promising tumor markers in breast cancer (review). Anticancer Res. 1996, 16, 2233-2239. [PubMed]

22. Na, M.; Cui, L.; Min, B.S.; Bae, K.; Yoo, J.K.; Kim, B.Y.; Oh, W.K.; Ahn, J.S. Protein tyrosine phosphatase 1B inhibitory activity of triterpenes isolated from Astilbe koreana. Bioorg. Med. Chem. Lett. 2006, 16, 3273-3276. [CrossRef] [PubMed]

23. Na, M.; Oh, W.K.; Kim, Y.H.; Cai, X.F.; Kim, S.H.; Kim, B.Y.; Ahn, J.S. Inhibition of protein tyrosine phosphatase 1B by diterpenoids isolated from Acanthopanax koreanum. Bioorg. Med. Chem. Lett. 2006, 16, 3061-3064. [CrossRef] [PubMed]

24. Burke, T.R., Jr.; Ye, B.; Yan, X.; Wang, S.; Jia, Z.; Chen, L.; Zhang, Z.Y.; Barford, D. Small molecule interactions with protein-tyrosine phosphatase PTP1B and their use in inhibitor design. Biochemistry 1996, 35, 15989-15996. [CrossRef] [PubMed]

Sample Availability: Samples of the compounds are available from the authors. A voucher specimen (No. 2014-04) has been deposited at the Herbarium of the Korea Bioactive Natural Material Bank, Seoul, Korea.

(C) 2016 by the authors; licensee MDPI, Basel, Switzerland. This article is an open access article distributed under the terms and conditions of the Creative Commons Attribution (CC-BY) license (http://creativecommons.org/licenses/by/4.0/). 